\title{
Innovare l'offerta estendendo il retail brand: il ruolo della fedeltà e della sensibilità a prezzo e promozione
}

\author{
Elisa MARTINELLI** BeAtRICE LUCERI ${ }^{* * *}$ SABRINA LATUSI****
}

\begin{abstract}
Obiettivo del paper: L'articolo indaga il ruolo che la fedeltà alla marca commerciale, la sensibilità al prezzo e quella alla promozione svolgono nel predire l'acquisto di un'offerta innovativa nel retail grocery, ovvero: i prodotti e servizi extra-core a marca insegna (PSEx).

Metodologia: Ai dati ottenuti tramite un'indagine campionaria in-store su questionario strutturato (N. 139) è stata applicata una regressione logistica binaria.

Risultati: La fedeltà comportamentale alla marca commerciale e l'attitudine alla promozione risultano predittori dell'estensione d'acquisto, mentre la sensibilità al prezzo è solo debolmente significativa.

Limiti della ricerca: Il campione indagato è relativo ad una sola insegna. Il concetto di fedeltà considerato rappresenta solo una, anche se necessaria, delle componenti in cui si articola la customer loyalty. L'analisi non distingue tra singoli prodotti/servizi extra-core.

Implicazioni pratiche: I risultati emersi consentono di supportare e dare validazione empirica alle scelte di estensione dell'offerta operate dai manager della distribuzione despecializzata. Implementando politiche volte ad incrementare la frequenza e lo scontrino d'acquisto della marca commerciale sull'assortimento core e investendo in offerte promozionali si innesca un processo di trascinamento virtuoso delle nuove tipologie di offerta extra-core. Per avere successo nella nuova offerta, $i$ distributori devono concentrare $i$ loro sforzi di marketing sui clienti più sensibili all'acquisto di prodotti a marca commerciale e alle promozioni below the line.

Originalità del lavoro: Il contributo verifica l'applicabilità di alcuni risultati emergenti dagli studi sulla marca commerciale anche ad un suo concetto esteso, ovvero in un contesto,
\end{abstract}

* Nonostante l'articolo sia frutto del lavoro congiunto delle autrici, può essere attribuito alle stesse così come di seguito specificato: Elisa Martinelli ha in particolare contribuito ai parr. 3, 5 e 6; Beatrice Luceri ai parr. 1 e 2; Sabrina Latusi ai parr. 4 e 7.

** Ricercatore di Economia e Gestione delle Imprese - Università degli Studi di Modena e Reggio Emilia

e-mail: elisa.martinelli@unimore.it

*** Associato di Economia e Gestione delle Imprese - Università degli Studi di Parma e-mail: beatrice.luceri@unipr.it

**** $\quad$ Ricercatore di Economia e Gestione delle Imprese - Università degli Studi di Parma e-mail: sabrina.latusi@unipr.it 
quello dei prodotti/servizi extra-core, innovativo e non ancora analizzato in modo approfondito dalla letteratura.

Parole chiave: marca commerciale; prodotti e servizi extra-core; fedeltà; prezzo; promozione

Purpose of the paper: The paper investigates the role that loyalty to the private label, value consciousness and promotion attitude exert in predicting the buying of non-traditional products and services offered with the retail brand name.

Methodology: A logistic binary regression was applied to the data collected through an in-store survey administering a structured questionnaire (N. 139).

Findings: Main findings evidence that behavioral loyalty to the private label and promotion attitude predict the buying of non-traditional retail products and services, while value consciousness is borderline.

Research limits: The sample collected concerns a sole retailer. The loyalty concept investigated represents only one of the necessary components of customer loyalty. The analysis does not distinguish within specific non-traditional products and services.

Practical implications: Results empirically support the innovative decision to extend the offer to no-core products and services by retail managers. Implementing policies aimed at increasing the purchase of private label within the core assortment and at investing in price promotions, non-traditional products and services could benefit from a virtuous cycle. To succeed in this new offer, retailers' efforts have to focus on the customers more sensitive to private label and below the line promotions.

Originality of the paper: The paper contributes to the literature on private label extending some of its results in the innovative context of non-traditional products and services, poorly explored since now.

Key words: private label; non-traditional products and services; loyalty; price; promotion

\section{Introduzione}

Il settore del grocery retailing è oggi caratterizzato da un acceso confronto competitivo che, soprattutto a causa della profonda crisi economica che attanaglia l'economia, si è spostato sempre di più verso una sottolineatura esasperata di un'immagine di convenienza relativa basata sul perseguimento di strategie di prezzo aggressive e, in particolare, su spinte politiche promozionali di tipo below the line. Veicolo importante nel perseguimento di questo obiettivo è l'offerta a marca commerciale, attualmente in crescita in tutta Europa. L'Associazione Internazionale dei Produttori di Marca Commerciale (PLMA, 2012) sostiene che i prodotti a marca commerciale hanno raggiunto quote del $40 \%$ e oltre in almeno i seguenti sei paesi europei: Svizzera (53\%), Spagna (49\%), Gran Bretagna (47\%), Portogallo (43\%), Germania (41\%) e Belgio (40\%).

La marca commerciale è però anche un'opportunità di innovazione per i retailer tramite cui rispondere a nuove esigenze o tendenze emergenti. Tra queste, la possibilità di soddisfare grappoli di bisogni estesi e complessi in condizioni di convenienza economica, logistica e cognitiva è certamente una possibile risposta ad un consumatore che ha rivisto le sue potenzialità di spesa ma che è spesso soggetto a 
time constraint e ricerca la semplificazione dei processi d'acquisto, soprattutto se routinari (Cristol e Sealey, 2000). Coprendo più esigenze, semplificandone la soddisfazione e aumentando i possibili punti di contatto con il cliente i distributori possono ottenere numerosi vantaggi, tra cui in particolare l'incremento di margini e vendite e la fidelizzazione della clientela. È in questa prospettiva che è possibile leggere la strategia di estensione dell'offerta che i distributori despecializzati operanti in Italia hanno iniziato a perseguire in tempi recenti. Nei punti vendita despecializzati è infatti oggi possibile acquistare parafarmaci, rifornirsi di carburante, sottoscrivere prodotti finanziari, piuttosto che prenotare spettacoli o prestazioni mediche/ambulatoriali, oltre ad approvvigionarsi di altre tipologie di possibili proposte, tutte a marca commerciale, molto lontane dal core business originario del comparto osservato - ovvero i prodotti di largo e generale consumo, cd. grocery. Si tratta di un'innovazione della proposta assortimentale dei distributori che apre la strada a diverse implicazioni sia nei rapporti con la domanda (Martinelli, 2010), sia a a livello competitivo (Martinelli, 2012).

In questo contesto, l'obiettivo che l'articolo si pone è quello di comprendere se e come la fedeltà comportamentale sviluppata dalla clientela verso i prodotti a marca commerciale e la sensibilità al prezzo e alla promozione vadano a predire l'acquisto di prodotti e servizi extra-core (PSEx). Dato che l'offerta di prodotti grocery a marca commerciale permette di incrementare il traffico e la fedeltà al punto vendita (Dick et al., 1995), verificare se chi acquista intensivamente prodotti a marca commerciale mostra anche una maggiore propensione all'acquisto di PSEx potrebbe portare ad una migliore comprensione delle potenzialità delle strategie di marca commerciale, anche in forma estesa, con interessanti ritorni dal punto di vista manageriale oltre che scientifico. $\mathrm{Ci}$ attendiamo infatti che siano i clienti più fedeli alla marca commerciale ad essere potenzialmente più sensibili a sottoscrivere $\mathrm{i}$ nuovi prodotti/servizi offerti. Egualmente, dato che la letteratura ha provato che gli acquirenti di prodotti a marca commerciale sono generalmente value conscious (Ailawadi et al., 2001), riteniamo interessante verificare l'impatto che la sensibilità al prezzo e alla promozione da parte della clientela possono esercitare sulle loro scelte di acquisto di PSEx.

Il conseguimento di questi obiettivi è operato tramite lo svolgimento di un'analisi quantitativa condotta su un campione di clienti di un punto vendita di un'insegna della Grande distribuzione Organizzata (GDO), leader del comparto a livello nazionale e fortemente coinvolta nell'offerta di prodotti e servizi non tradizionali, tramite la somministrazione di un questionario strutturato. I dati così raccolti sono poi stati oggetto di un'analisi di regressione logistica binaria.

L'articolo, dopo una breve rassegna della letteratura sull'innovazione della marca commerciale e sull'estensione d'offerta, presenta le ipotesi ed il modello che si intende testare. Segue la descrizione della metodologia utilizzata, partendo dall'operativizzazione dei costrutti e delle modalità di raccolta dati per poi presentare le caratteristiche del campione rilevato e delle tipologie, numerosità e frequenza d'acquisto dei PSEx osservati. L'illustrazione dei risultati emersi precede la loro discussione che evidenzia le implicazioni teoriche e manageriali emergenti 
dallo studio. L'articolo termina con alcune considerazione conclusive che indicano $\mathrm{i}$ limiti del lavoro e le linee prospettiche di ricerca che ne possono derivare.

\section{Innovare l'offerta con i prodotti/servizi extra-core a marca commerciale}

L'innovazione nel retail è stata tradizionalmente studiata in termini di innovazione di prodotto e quindi avendo a riguardo il prodotto commerciale in termini di forma distributiva e distinguendo le innovazioni tra primarie e secondarie (Spranzi, 1991). Le prime sono rare e volte a colmare vuoti d'offerta con trasformazioni radicali del servizio offerto e delle tecniche di produzione, dando così vita a nuove forme distributive e quindi a un nuovo settore distributivo (Lugli, 1993); le seconde sono maggiormente frequenti e si sostanziano in una riformulazione del servizio offerto da una certa forma distributiva preesistente e quindi avvengono all'interno del formato. Originariamente, la letteratura ha posto particolare attenzione alle dinamiche di sviluppo delle forme distributive grazie alle teorie del ciclo evolutivo quali la wheel of retailing (McNair, 1958; Hollander, 1960) e la retail life cycle (Davidson et al., 1976). Attenzione è stata posta ai fattori alla base dell'innovazione di formato, riconoscendo il ruolo critico svolto dalla competizione (Gist, 1968) e dalla domanda (Filser, 1992). Questa visione dell'innovazione non consente però di apprezzare la complessità e la varietà di contesti in cui la stessa si esprime in ambito retail. Lugli (1998) declina il tema avendo a riferimento la catena del valore distributivo e distinguendone le tipologie a seconda che l'innovazione impatti sul contenuto (assortimento e servizi offerti), sul contesto (merchandising) e/o sull'infrastruttura (grado e modalità di coinvolgimento sia dell'acquirente che del fornitore nella erogazione del servizio commerciale). Dupuis (2001) categorizza le innovazioni nel retailing in quattro principali tipologie:

- le innovazioni di concetto, relative alle modalità di erogazione del servizio e di interfacciamento con la domanda;

- le innovazioni di flusso, riguardanti modalità nuove di gestione delle relazioni verticali al fine di accrescere l'efficienza nello svolgimento degli scambi logistici e informativi;

- le innovazioni organizzative, volte a migliorare le modalità di gestione delle relazioni intra- e inter-organizzative;

- le innovazioni architettoniche, determinate da ricombinazioni delle precedenti tipologie.

Castaldo e Mauri (2007) evidenziano in particolare tre forme di innovazione nella distribuzione commerciale: technology-push, market-based, di canale. L'impatto dell'innovazione nel retail riguarda pertanto sia i processi gestionali, sia l'articolazione dell'offerta, sia la struttura della value proposition (Dawson, 2001). Nel presente contributo l'analisi si concentra su questa terza direttrice di innovazione, considerando una prospettiva di arricchimento della value proposition tramite l'offerta di categorie di prodotto/servizio "inusuali" rispetto alla tradizionale 
offerta di punti vendita despecializzati. Le imprese della GDO, oltre a puntare sulla convenienza dell'offerta, area di imprescindibile presidio nel grocery, necessitano infatti di differenziarsi attraverso la componente funzionale, esperienziale e valoriale dei bisogni soddisfatti. In questa prospettiva, l'estensione del core service a categorie extra può acquisire la natura di servizio interno (Botti e Soscia, 1999) - se intrinsecamente connesso al core service, senza cui non potrebbe sussistere (ad esempio: l'offerta di servizi di ristorazione) - o di servizio esterno (Bolton e Drew, 1994) - se indipendente dall'attività principale di logistica ed assortimento svolta dai retailer (ad esempio: i servizi finanziari e turistici).

Busacca e Bertoli (2012) evidenziano come le strategie di brand extension tramite l'ingresso in nuove aree di business vadano ad incidere sia sulle opportunità di generazione di flussi di risultato incrementali, sia sullo sviluppo delle risorse di conoscenza dell'impresa, determinando un ampliamento e approfondimento della rete di relazioni con la domanda. Ne deriva che la strategia di estensione della marca può essere impiegata per conseguire un duplice obiettivo: ampliare il portafoglioclienti e rafforzare la fedeltà dei clienti attuali.

Dal contributo di Myers (1967), la letteratura di marketing e di retail ha evidenziato un crescente interesse per lo studio della marca commerciale, rafforzato $\mathrm{e}$ giustificato nel tempo dal costante incremento della quota di mercato da essa sviluppata. Si tratta infatti di una politica che impatta fortemente sulle relazioni di mercato che l'impresa di distribuzione commerciale intrattiene a monte, a valle e con i concorrenti.

In Italia la marca commerciale nasce e si sviluppa con l'affermazione della grande distribuzione (Pastore, 1996). Ciò comporterà una nuova fonte di conflittualità nei rapporti tra produttore e distributore (Varaldo e Dalli, 1989), che non solo si troveranno ad essere competitor diretti sugli scaffali dei punti vendita moderni, ma inizieranno a contendersi le preferenze della clientela finale anche in termini di relazione di lungo periodo, andando a configurare una situazione in cui "la fidelizzazione alla marca industriale o alla marca commerciale e all'insegna rappresenterà la vera posta in gioco" (Golinelli, 2004, p. 222).

Da allora la marca commerciale ha accresciuto la sua penetrazione, divenendo fattore cruciale di competitività delle insegne distributive (Lugli, 2003).

Le motivazioni alla base della crescita della marca commerciale possono essere identificate principalmente nelle seguenti: innanzitutto, il crescente livello di concentrazione delle imprese di distribuzione al dettaglio, in quanto l'offerta a marca commerciale richiede economie di scala per essere efficientemente conseguita; secondo, un atteggiamento del consumatore maggiormente positivo, in generale, verso questi prodotti grazie ad un innalzamento del loro livello qualitativo (Steenkamp et al., 2010); terzo, il livello di convenienza relativo offerto rispetto ai prodotti a marca industriale, fattore particolarmente importante durante la fase di crisi economica che sta attraversando il paese e che contribuisce a rendere la marca commerciale una referenza d'acquisto consolidata nei panieri d'acquisto della domanda. 
La competitività della marca commerciale è principalmente legata alla convenienza di prezzo e ai minori costi di produzione, packaging e comunicazione che in genere questi prodotti hanno rispetto a quelli di marca industriale (Cunningham et al., 1982; Baltras, 1997; Putsis e Dhar, 2001). Ciò ha comportato riflessi non solo dal punto di vista della competizione orizzontale, ma anche verticale, nei confronti in primis dell'industria di marca verso cui la distribuzione ha incrementato il proprio potere contrattuale e di mercato (Ailawadi e Keller, 2004; Karry e Zaccour, 2006; Lugli, 2009) ed oggi anche per le interessanti sfide trasversali supportate dalla convergenza inter-industry che l'ampliamento dell'offerta a categorie extra-core apre (Martinelli, 2012).

Dal punto di vista della domanda, i prodotti a marca commerciale sono in genere percepiti come di qualità inferiore rispetto a quelli di marca industriale (Richardson et al., 1994; Sprott e Shimp, 2004). Tale atteggiamento si sta però progressivamente modificando anche grazie alle politiche di riposizionamento perseguite dalla distribuzione (Pastore et al., 2007). I benefici che la clientela può ottenere acquistando prodotti a marca commerciale sono da individuare prima di tutto nel differenziale di prezzo rispetto ai prodotti a marca industriale. Inoltre, la marca commerciale consente un ampliamento dell'offerta assortimentale disponibile al consumatore, sia in ampiezza che in profondità, ed un'intensificazione dell'attività promozionale (Pauwels e Srinivasan, 2004).

In sintesi, la marca commerciale consente ai distributori di ottenere diversi vantaggi di tipo strategico. Innanzitutto, offrire prodotti a marca commerciale permette di incrementare il traffico (Dick et al., 1995), e la fedeltà al punto vendita (Cortjens e Lal, 2000; Dekimpe et al., 1997), di incrementare la marginalità e il proprio potere contrattuale nei confronti dei fornitori industriali (Lugli, 2009; Ailawadi et al., 2008). Inoltre, la marca commerciale consente di differenziarsi dai competitor (Sprott e Shimp, 2004), e permette di godere di maggiore flessibilità nella definizione delle politiche di prezzo e promozionali (Nogales e Suares, 2005).

Seppure attestata su livelli più contenuti rispetto a quanto accade in altri contesti distributivi europei (UK; Francia; Germania), la marca commerciale sta registrando in Italia un interessante trend di incremento, raggiungendo una quota di mercato del 18,1\% nel 2012 (SymphonyIRI Group, 2013). Nel tempo, i distributori hanno esteso il numero di merceologie in cui sono presenti prodotti a marca commerciale, oggi offerti nel $91 \%$ delle categorie. Il crescente livello di competizione presente nel settore distributivo grocery e la tendenziale saturazione delle potenzialità di crescita sul mercato interno non fanno che rafforzare l'orientamento ad ampliare la proposta assortimentale, anche ad un crescente numero di categorie non-food e di servizi, anche distanti dal core-business originario (Burt, 2000; Colgate e Alexander, 2002). L'estensione delle categorie di prodotto offerte anche ad ambiti extra-grocery e ai servizi è quindi diventato un obiettivo strategico per le imprese del settore (Laforet, 2007). 


\section{Le ipotesi della ricerca}

La ricerca presentata in questo articolo si propone di verificare l'influenza che una serie di fattori, ovvero la fedeltà comportamentale alla marca commerciale, l'attenzione al prezzo e l'atteggiamento nei confronti delle iniziative promozionali di taglio prezzo, esercitano sull'acquisto di PSEx. Nel presente paragrafo si andranno pertanto ad enunciare le ipotesi di ricerca.

La letteratura ha da tempo riconosciuto la valenza strategica dei clienti fedeli alla marca (Reichheld, 1993): si tratta infatti di soggetti più propensi ad acquistare nuovi prodotti (Reichheld e Sasser, 1990) e ad ampliare la relazione fiduciaria con la marca all'intera gamma di beni proposti con questo brand (Grayson e Ambler, 1999). In accordo infatti alla teoria della categorizzazione (Kahneman e Tversky, 1973), da cui si sono poi sviluppati gli studi sulla brand extension (Keller, 1993), l'estensione della marca a nuove categorie di prodotto beneficia dell'effetto positivo dell'associazione con il brand (Aaker e Keller, 1990; Park et al., 1991). Diversi autori hanno pertanto concentrato la loro attenzione sull'effetto che la consonanza percettiva (perceptual fit) esercita ai fini del successo dell'estensione. Bertoli et al. (2008) hanno ad esempio verificato che la consonanza percettiva risulta essere la variabile che esercita il maggiore impatto diretto e positivo sull'atteggiamento verso l'estensione, seguita dalla brand reputation e dalla brand familiarity.

Nel presente studio si considera una concettualizzazione di tipo comportamentale della fedeltà, ovvero riferita al comportamento d'acquisto ripetitivo del cliente. In questo senso, la fedeltà del cliente è stata rappresentata tramite misuratori dell'attività di acquisto ripetuto e/o tramite la valutazione della durata della relazione nel tempo. È su questo concetto che una parte consistente degli studi che indagano il tema della fedeltà in contesti distributivi si è focalizzato (Huddleston $e t$ al., 2004; Mägi, 2003) operativizzando il costrutto tramite indicatori di percentuale di spesa in un certo punto vendita (Macintosh e Lockshin, 1997; Sirohi et al., 1998), ammontare speso (Corstjens e Lal, 2000), frequenza di visita (De Wulf et al., 2001; Mägi, 2003).

Possiamo pertanto ipotizzare che coloro che acquistano frequentemente e che destinano un consistente ammontare di risorse all'acquisto di prodotti a marca commerciale siano anche i clienti maggiormente disposti ad acquistare i nuovi prodotti/servizi extra-core offerti dalle insegne distributive grocery.

HP1: la fedeltà comportamentale nei confronti della marca commerciale predice l'acquisto di prodotti/servizi extra-core

In generale, la letteratura ha evidenziato che gli acquirenti di prodotti a marca commerciale tendono ad essere maggiormente price sensitive (Dick et al., 1995). Sethuraman (2003), nella sua rassegna degli studi sul tema, evidenzia che ben 19 contributi su 23 riportano dell'importanza del prezzo nell'influenzare le vendite dei prodotti a marca commerciale. Gli acquirenti di prodotti a marca commerciale sono generalmente value conscious (Ailawadi et al., 2001) e tendono a ricercare convenienza di prezzo. Secondo Lichtenstein et al. (1993, p. 235) "value 
consciousness is a concern for paying low prices subject to some quality constraints". I clienti di questo tipo si contraddistinguono pertanto per l'attitudine a massimizzare il rapporto qualità/prezzo quando effettuano acquisti.

Una serie di studi (Richardson et al., 1994; Batra e Sinha, 2000; Ailawadi et al., 2001) si sono specificamente diretti ad investigare la forza della relazione tra sensibilità al prezzo della domanda e intenzione d'acquisto della marca commerciale piuttosto che l'atteggiamento nei confronti della marca commerciale. In questa prospettiva la marca commerciale è stata riconosciuta come strumento che aiuta i retailer a competere con profitto nel segmento sensibile al prezzo (Corstjens e Lal, 2000). E dato che le evidenze emerse in uno studio esplorativo (Martinelli, 2010) mettevano in evidenza la convenienza di prezzo dell'offerta come la principale motivazione d'acquisto di PSEx possiamo così di seguito formulare la seconda ipotesi che il presente studio si propone di verificare.

HP2: I clienti maggiormente sensibili al prezzo sono maggiormente inclini ad acquistare prodotti/servizi extra-core.

I prodotti a marca commerciale sono oggi soggetti ad una forte pressione promozionale. Si tratta di una prassi che si discosta da quanto suggerito dalla letteratura (Lugli, 2009) che in virtù della ridotta elasticità della domanda al prezzo, dell'assenza dei contributi promozionali dei fornitori e dell'orientamento del retailer al marketing di categoria anziché di prodotto non supporterebbe un uso intensivo della politica promozionale per la marca commerciale. La pratica operativa evidenzia però tutt'altro scenario: la pressione promozionale sulla marca privata nel 2011 si è attestata al 20,9\%, in aumento rispetto a ciò che avviene per i brand industriali, con una presenza sul volantino in deciso incremento di 4 punti percentuali rispetto al 2007 (Nielsen Trade*Mis, 2012). Possiamo pertanto ipotizzare che i clienti più sensibili alle promozioni siano anche quelli più propensi ad acquistare le nuove offerte non-food e di servizio a cui viene estesa la private label.

HP3:I clienti che mostrano un atteggiamento positivo nei confronti delle promozioni di prezzo sono maggiormente inclini ad acquistare prodotti/servizi extra-core.

\section{Metodologia}

\subsection{Operativizzazione dei costrutti}

I costrutti indagati sono stati desunti da scale testate in letteratura (Tab. 1). Gli item impiegati hanno utilizzato misurazioni su scala Likert 1-7 (1= completamente in disaccordo; $7=$ completamente d'accordo). 
Tab. 1: Costrutti indagati e item impiegati

\begin{tabular}{|c|c|c|}
\hline Costrutti & Item & Riferimenti \\
\hline \multirow{2}{*}{$\begin{array}{l}\text { Fedeltà } \\
\text { comportamentale } \\
\text { alla marca } \\
\text { commerciale } \\
\text { (BLMC) }\end{array}$} & Frequenza d'acquisto di prodotti a marca commerciale & \multirow{2}{*}{$\begin{array}{l}\text { Corstjens e } \\
\text { Lal 2000; } \\
\text { Mägi } 2003\end{array}$} \\
\hline & $\%$ di reddito speso in prodotti a marca commerciale & \\
\hline \multirow{7}{*}{$\begin{array}{l}\text { Attenzione al } \\
\text { prezzo (ATTPR) }\end{array}$} & $\begin{array}{l}\text { Presto molta attenzione ai prezzi bassi, ma sono } \\
\text { ugualmente attento anche alla qualità del prodotto }\end{array}$ & \multirow{7}{*}{$\begin{array}{l}\text { Burton et } \\
\text { al. 1998; } \\
\text { Lichtenstein } \\
\text { et al. 1993, } \\
\text { Bao et al., } \\
2011\end{array}$} \\
\hline & $\begin{array}{l}\text { Quando faccio la spesa al supermercato, comparo i } \\
\text { prezzi delle differenti marche per essere sicuro di } \\
\text { ottenere il miglior rapporto qualità/prezzo }\end{array}$ & \\
\hline & $\begin{array}{l}\text { Quando acquisto un prodotto, cerco sempre di } \\
\text { ottimizzare la qualità ottenibile per il prezzo pagato }\end{array}$ & \\
\hline & $\begin{array}{l}\text { In genere, mi guardo intorno alla ricerca dei prezzi più } \\
\text { bassi dei prodotti, ma questi devono comunque } \\
\text { mantenere certi requisiti qualitativi affinché io li acquisti }\end{array}$ & \\
\hline & $\begin{array}{l}\text { Quando faccio acquisti, in genere confronto il prezzo/kg } \\
\text { dei prodotti rispetto alle marche che acquisto di solito }\end{array}$ & \\
\hline & $\begin{array}{l}\text { Quando acquisto i prodotti, mi piace essere sicuro di } \\
\text { aver speso beni i miei soldi }\end{array}$ & \\
\hline & $\begin{array}{l}\text { Controllo sempre i prezzi in punto vendita in modo da } \\
\text { assicurarmi di ottenere il maggior valore possibile per il i } \\
\text { soldi che spendo }\end{array}$ & \\
\hline \multirow{3}{*}{$\begin{array}{l}\text { Attitudine alla } \\
\text { promozione } \\
\text { (ATTPROMO) }\end{array}$} & Acquistare marche in offerta mi rende felice & \multirow{3}{*}{$\begin{array}{l}\text { Garretson } \\
\text { et al. 2002; } \\
\text { Manzur et } \\
\text { al. } 2011\end{array}$} \\
\hline & $\begin{array}{l}\dot{E} \text { più probabile che io acquisti marche che sono in } \\
\text { offerta promozionale piuttosto che marche non scontate }\end{array}$ & \\
\hline & Mi appaga acquistare una marca in offerta & \\
\hline
\end{tabular}

Fonte: ns. elaborazioni

Il questionario così definito è prima stato testato al $10 \%$ per poi essere somministrato su base estensiva all'uscita della barriera casse di un punto vendita della principale insegna operante nella distribuzione grocery a livello nazionale, sia per dimensione che per attuale coinvolgimento nell'offerta di prodotti/servizi extracore. La scelta di un ambito di indagine reale risponde all'intento di dare maggiore solidità alle informazioni raccolte.

La prima domanda posta agli intervistati è stata volta a comprendere se i clienti dell'insegna indagata avessero acquistato PSEx negli ultimi sei mesi e nel caso di risposta affermativa si richiedeva agli stessi di indicare quale tipologia avessero scelto tra quelle osservate, ovvero: parafarmacia, sviluppo foto, telefonia mobile, prenotazione viaggi, servizi finanziari, prenotazione spettacoli, pagamento utenze, prenotazione CUP.

Un campione di convenienza complessivo di 139 interviste è stato quindi raccolto su un arco temporale bisettimanale, avendo cura di predisporre un piano di rilevazione suddiviso in diverse fasce orarie della giornata in modo da raccogliere la maggior varietà possibile di profili di clientela e di modelli d'acquisto. 


\subsection{Le caratteristiche del campione}

Il campione è costituito per l' $87,1 \%$ da acquirenti donne.

Il $69,1 \%$ del campione è rappresentato da persone in età matura. Il segmento maggiormente presente è, infatti, quello dei rispondenti nella classe di età 36 - 50 anni (39,6\% del campione), seguito da coloro che hanno un'età compresa tra i 51 ed i 65 anni $(29,5 \%)$. Minimale $(2,9 \%)$ la fascia dei giovanissimi, mentre di peso simile è la presenza di giovani $(14,4 \%)$ e anziani $(13,7 \%)$.

Il livello di istruzione degli intervistati è buono: il $44,6 \%$ possiede il diploma di scuola superiore, mentre il $30,2 \%$ ha conseguito il diploma di laurea o di post laurea, con prevalenza del primo $(16,5 \%)$. Seguono coloro che possiedono il diploma di scuola media inferiore $(19,4 \%)$, mentre solamente il $5,8 \%$ ha la licenza elementare.

La composizione familiare evidenzia nuclei abbastanza variegati, composti prevalentemente da $4(30,9 \%), 3(28,8 \%)$ o $2(23 \%)$ persone. Ridotta la presenza di mono - famiglie $(9,4 \%)$ e di nuclei familiari composti da 5 o più persone $(7,9 \%)$.

Il reddito complessivo dei nuclei familiari, infine, è medio - basso: il 48,2\% degli intervistati dichiara di rientrare nella fascia di reddito compresa tra 36.000 e 70.000 euro, mentre il $42,4 \%$ possiede un reddito inferiore a 36.000 euro. Seguono coloro che si collocano nella fascia di reddito compresa tra 70.000 e 100.000 euro $(7,2 \%)$, mentre è esigua la percentuale di coloro il cui reddito supera i 100.000 euro $(2,2 \%)$.

\subsection{I prodotti/servizi extra-core acquistati}

L'indagine ha innanzitutto accertato se i consumatori che si recano a fare la spesa nel punto vendita osservato sono soliti acquistare i prodotti e servizi extra offerti dall'insegna, raccogliendo risultati decisamente differenziati in funzione della categoria offerta (Tab. 2). Un'elevata percentuale di intervistati $(65,5 \%)$ ha dichiarato di acquistare parafarmaci nel corner di parafarmacia a marca insegna presente in-store. L'offerta di sviluppo foto e di telefonia mobile ha visto l'attivazione del servizio da parte rispettivamente del $28,1 \%$ e del $19,4 \%$ del campione. Percentuali simili si sono registrate, invece, per i servizi di pagamento delle utenze in cassa $(12,9 \%)$ e di prenotazione dei viaggi $(11,5 \%)$. Infine, i servizi finanziari, la prenotazione CUP e la possibilità di prenotare spettacoli in punto vendita hanno ottenuto uno scarso riscontro nei consumatori intervistati.

Rispetto alla frequenza di acquisto, la parafarmacia è il prodotto/servizio extra che viene acquistato più frequentemente, pur se su livelli medi contenuti $(3,66$ in media), seguito dalla telefonia mobile e dallo sviluppo di fotografie digitali. Tutti gli altri servizi erogati dall'insegna commerciale sono acquistati in modo occasionale. 
Tab. 2: Le tipologie dei prodotti/servizi extra-core acquistati

\begin{tabular}{|l|c|c|c|c|}
\hline Tipologia di PSEx & \multicolumn{2}{|c|}{ Acquirenti } & \multicolumn{2}{c|}{ Frequenza d'acquisto * } \\
\hline & N. & $\%$ & Media & Dev. std. \\
\hline Parafarmaci nel corner & 91 & 65,5 & 3,66 & 2,391 \\
\hline Sviluppo foto digitali & 39 & 28,1 & 1,86 & 1,666 \\
\hline Telefonia mobile & 27 & 19,4 & 2,01 & 2,157 \\
\hline Prenotazione viaggi & 16 & 11,5 & 1,41 & 1,340 \\
\hline Servizi finanziari & 13 & 9,4 & 1,47 & 1,529 \\
\hline Prenotazione spettacoli & 4 & 2,9 & 1,09 &, 558 \\
\hline Pagamento utenze in cassa & 18 & 12,9 & 1,57 & 1,556 \\
\hline Prenotazione CUP & 10 & 7,2 & 1,27 & 1,047 \\
\hline
\end{tabular}

Fonte: ns. elaborazioni

Coloro che comunque hanno acquistato almeno un PSEx tra quelli osservati sono il $74,1 \%$ del campione (Tab. 3).

Tab. 3: La suddivisione del campione in base all'acquisto di PSEx

\begin{tabular}{|l|c|c|}
\hline & Frequenza & $\%$ \\
\hline Non hanno acquistato PSEx & 36 & 25,9 \\
\hline Hanno acquistato PSEx & 103 & 74,1 \\
\hline Totale campione & 139 & 100,0 \\
\hline
\end{tabular}

Fonte: ns. elaborazioni

\section{I risultati}

I costrutti osservati presentano valori sopra la media di scala (Tab. 4). Si tratta di acquirenti del punto vendita che scelgono la marca commerciale abbastanza di frequente e vi destinano un discreto ammontare di risorse $(M=4,33)$ e che si dichiarano particolarmente attenti alla convenienza di prezzo di ciò che acquistano $(\mathrm{M}=5,88)$ e alle offerte promozionali $(\mathrm{M}=5,74)$.

Tab. 4: I predittori: valori medi e deviazione standard

\begin{tabular}{|l|c|c|c|}
\hline Predittori & $N$. & Media & Deviazione std. \\
\hline BLMC & 139 & 4,3345 & 1,16779 \\
\hline ATTPR & 139 & 5,8801 & 1,01380 \\
\hline ATTPROMO & 139 & 5,7446 & 1,44258 \\
\hline
\end{tabular}

Fonte: ns. elaborazioni

L'affidabilità dei costrutti osservati è ben superiore al valore soglia raccomandato di 0.70 (Nunnally e Bernstein, 1994) e si qualifica come molto elevata ( $\alpha$ BLMC: $0.87 ; \alpha$ ATTPR: 0.90; $\alpha$ ATTPROMO: 0.90). Solo un item è stato eliminato relativamente al costrutto dell'attitudine alla promozione. L'analisi 
fattoriale che è successivamente stata svolta ha confermato l'unidimensionalità dei costrutti impiegati, ad ulteriore conferma della loro affidabilità.

Una regressione logistica binaria è stata a questo punto condotta, impiegando la variabile relativa all'acquisto di almeno un $(\mathrm{N}=1)$ PSEx come variabile dipendente $(1=$ acquisto; $0=$ non acquisto $)$ ed $\mathrm{i}$ tre costrutti sopra evidenziati come variabili indipendenti. La scelta di questa tecnica statistica di elaborazione dei dati raccolti deriva dalla sua particolare attitudine ad essere impiegata in letteratura per indagare le decisioni di acquisto o non acquisto relative al processo decisionale del consumatore (Akinci et al., 2007).

Il modello presenta indicatori buoni e significativi, mostrando la sua idoneità ad essere adattato ai dati raccolti $\left[\mathrm{R}^{2}=6.6\right.$ (Hosmer e Lemeshow, 2000), 0.08 (Cox e Snell, 1989), 0.12 (Nagelkerke, 1991). Model $\left.\chi^{2}(3)=11.08, p<0,01\right]$. Relativamente ai test diagnostici, le statistiche dei residui sono buone, in quanto:

- i residui standardizzati sono sostanzialmente compresi nell'intervallo \pm 2 : meno dell' $1 \%$ dei casi supera infatti il limite soglia di $\pm 2,5$;

- tutti i casi hanno DFbeta minore di 1;

- l'indicatore della distanza di Cook non presenta nessun valore sopra 1.

In conclusione, non sono presenti casi peculiari che possano influenzare il modello testato.

Relativamente alla verifica di una possibile presenza di multicollinearità, i valori di tolleranza sono risultati essere ben sopra al valore soglia di 0.1 (Menard, 1995), mentre la statistica VIF risulta ben al di sotto del valore 10 (Myers, 1990), confermando che i predittori impiegati non sono affetti da problemi di multicollinearità, così come evidenziato anche dalla diagnostica di collinearità.

In sintesi, i risultati emersi dall'analisi di regressione logistica binaria svolta (Tab. 5) evidenziano che la fedeltà comportamentale alla marca commerciale e l'attitudine alla promozione sono predittori dell'acquisto di PSEx, mentre la sensibilità al prezzo è debolmente significativa $(\mathrm{p}<0,1)$.

Tab. 5: Le relazioni tra le variabili indagate

\begin{tabular}{|l|c|c|c|c|}
\hline & & \multicolumn{3}{|c|}{$95 \% \operatorname{Cl} \operatorname{per} \operatorname{Exp}(\beta)$} \\
\hline Costante & $\mathrm{B}(\mathrm{es})$ & Inferiore & $\operatorname{Exp}(\mathrm{B})$ & Superiore \\
\hline BLMC & $-0,14(1,34)$ & & & \\
\hline ATTPROMO & $0,49^{*}(0,18)$ & 1,143 & 1,632 & 2,330 \\
\hline ATTPRE & $0,34^{\star *}(0,17)$ & 1,012 & 1,409 & 1,962 \\
\hline
\end{tabular}

${ }^{*} \mathrm{p}<0,01$

$* * p<0,05$

$* * * p<0,1$

Fonte: ns. elaborazioni

Inoltre, i due predittori significativi evidenziano una relazione diretta con l'acquisto di PSEx: all'aumentare della fedeltà comportamentale alla marca commerciale o dell'attitudine ad acquistare in promozione, la probabilità di acquistare PSEx aumenta (odds ratio>1). È in particolare la fedeltà comportamentale 
alla marca commerciale che esercita l'effetto predittivo più forte rispetto all'acquisto della proposta estesa, in quanto capace di spiegare circa metà della decisione di acquisto. L'attenzione al prezzo, che assume valori di significatività borderline, mostra invece una relazione negativa con l'acquisto di PSEx, ovvero: maggiore l'attenzione al prezzo da parte della clientela, minore la probabilità che la stessa scelga le nuove tipologie di offerta proposte dal distributore.

\section{Le implicazioni teoriche e manageriali dello studio}

Le evidenze emerse dalla ricerca ci permettono di affermare che avere clienti fedeli dal punto di vista comportamentale alla marca commerciale consente di incrementare le probabilità di successo che i retailer grocery possono ottenere nell'estensione d'offerta a categorie anche molto lontane dal core business. L'HP1 è infatti verificata.

L'analisi ha inoltre evidenziato che i clienti che mostrano un atteggiamento positivo nei confronti delle promozioni di prezzo sono maggiormente inclini ad acquistare prodotti/servizi extra-core, dando così supporto empirico anche all'HP3.

Diversamente, i risultati emersi non consentono di evidenziare una forte influenza predittiva e positiva della value consciousness rispetto all'acquisto di prodotti/servizi extra-core, così come invece ci attendevamo. Questo risultato è da valutare con cautela visto che l'attenzione al prezzo è risultata avere valori di significatività borderline, ma crediamo possa essere interessante riflettere sul suo impatto negativo sull'acquisto di PSEx. Tale evidenza può essere probabilmente spiegata con il fatto che queste categorie di offerta extra-core non vengono acquistate nei punti vendita della distribuzione despecializzata in virtù del loro prezzo attrattivo, quanto primariamente per la convenienza logistica e cognitiva che deriva dal fatto di potere dare risposta a grappoli di vari bisogni in un'unica shopping expedition.

Le evidenze ottenute consentono pertanto di estendere alcune considerazioni che emergono dagli studi sulla marca commerciale anche ad un suo concetto esteso, e quindi in un contesto, quello dei prodotti/servizi extra-core, che non è ancora stato analizzato in modo approfondito dalla letteratura. In questo senso la ricerca contribuisce ad accrescere il valore che l'investimento in politiche volte a fidelizzare il cliente alla marca commerciale comporta.

Lo studio di Corstjens e Lal (2000) sulla costruzione della store loyalty attraverso lo sviluppo di store brand, dimostra che i consumatori che acquistano prodotti a marchio del distributore, in più categorie, saranno i meno inclini a visitare nuovi punti di vendita nelle future spedizioni di acquisto, in quanto il costo di effettuare una brand-switching nelle molteplici categorie risulterebbe troppo elevato. Le evidenze emerse dal presente contributo sono coerenti con questa prospettiva. $\mathrm{Ne}$ deriva che l'estensione d'offerta a categorie extra-core effettuato tramite il sostegno della marca commerciale è una strategia vincente in termini di fidelizzazione più complessiva del cliente al punto vendita. 
I risultati emersi possono pertanto essere utili ai manager della distribuzione despecializzata per trovare supporto e validazione empirica alle recenti scelte di estensione dell'offerta, perseguite spesso in realtà in modo sperimentale e non sempre in ottica strategica. Implementando politiche volte ad incrementare la frequenza e lo scontrino d'acquisto della marca commerciale sull'assortimento core e continuando ad investire in offerte promozionali si innesca infatti un processo di trascinamento virtuoso delle nuove tipologie di offerta extra-core. Sono quindi i clienti più sensibili all'acquisto di prodotti a marca commerciale e alle promozioni below the line su cui si dovrebbero concentrare gli sforzi dei distributori per avere successo nell'estensione d'offerta. Segmentando la clientela sulla base di questi due indicatori e sfruttando il potenziale strategico dei sistemi di CRM collegati alle carte fedeltà, i retailer potrebbero ottimizzare il ritorno sull'investimento effettuato nell'ampliamento dell'offerta.

\section{Considerazioni conclusive e limiti del lavoro}

Lo studio ha evidenziato come la fedeltà comportamentale alla marca commerciale e l'attitudine alla promozione siano predittori dell'estensione d'acquisto, mentre la sensibilità al prezzo sia debolmente significativa. Verificando impatto e direzione di alcuni importanti fattori alle basi delle scelte d'acquisto, in termini estesi, della clientela, l'articolo contribuisce alla letteratura sulla marca commerciale e sulla brand extension, fornendo supporto empirico alle scelte di diversificazione assortimentale operate dai distributori grocery.

Nonostante il contributo teorico e manageriale emergente dai risultati dell'analisi, alcuni limiti sono presenti nello studio che la ricerca futura potrebbe cercare di superare.

Innanzitutto, il campione di indagine potrebbe essere esteso numericamente per rafforzare la solidità delle relazioni evidenziate, soprattutto considerando la debole significatività della sensibilità al prezzo. Si potrebbe poi ripetere la rilevazione in diverse aree geografiche, così da cogliere eventuali difformità di comportamento dei consumatori residenti nelle tre principali macro-aree in cui si suddivide il paese (nord, centro, sud/isole).

Inoltre, l'analisi sul campo è stata svolta avendo a riferimento una sola insegna distributiva ed un solo punto vendita. Ciò non esclude una eventuale presenza di specificità che potrebbero avere influito sui risultati ottenuti. Obiettivo della ricerca futura potrebbe pertanto essere quello di estendere l'indagine ad altre insegne e formati distributivi, così da tenere sotto controllo il differente posizionamento strategico dei retailer ed il diverso livello di servizio offerto da distinte tipologie di formato (ad esempio: supermercato vs ipermercato), pur nella consapevolezza della crescente convergenza tra formati oggi in atto. L'estensione a diverse insegne potrebbe inoltre consentire di effettuare un benchmark tra marche commerciali di cui tenere conto nell'analisi, con conseguente ritorno informativo di tipo competitivo di sicuro interesse manageriale. 
Ancora, il concetto di fedeltà qui considerato rappresenta solo una, anche se certamente necessaria, delle componenti in cui si articola il costrutto della customer loyalty. Verificare l'eventuale impatto di determinanti cognitive e affettive della fedeltà alla marca commerciale consentirebbe certamente una visione più completa del fenomeno analizzato.

Infine, l'analisi non ha distinto tra i prodotti/servizi extra-core osservati, mentre sono evidenti le specificità che li caratterizzano e che potrebbero comportare un peso diverso dei predittori impiegati per l'analisi.

\section{Bibliografia}

AAKER D.A., KELLER K.L. (1990), “Consumer Evaluations of Brand Extensions”, Journal of Marketing, vol. 54, n. 1, pp. 27-41.

AKINCI S., KAYNAK E., ATILGAN E., AKSOY S. (2007), "Where does the logistic regression analysis stand in marketing literature? A comparison of the Market Positioning of Prominent Marketing Journals", European Journal of Marketing, vol. 41, n. 5, pp. 537-567.

AILAWADI K.L., PAUWELS K., STEENKAMP J.B. (2008), "Private-label use and store loyalty", Journal of Marketing, vol. 72, n. 6, pp. 19-30.

AILAWADI K.L., KELLER L.K. (2004), "Understanding retail branding: conceptual insights and research priorities", Journal of Retailing, vol. 80, n. 4, pp. 331-342.

AILAWADI K.L., NESLIN S.A., GEDENK G. (2001), "Pursuing the value-conscious consumer: store brands versus national brand promotions", Journal of Marketing, vol. 65, n. 1, pp. 71-89.

BALTRAS G. (1997), "Determinants of store brand choice: a behavioral analysis", Journal of Product Brand Management, vol. 6, n. 5, pp. 315-324.

BAO Y., BAO Y., SHENG S. (2011), "Motivating Purchase of Private Brands: Effects of Store Image, Product Signatureness, and Quality Variation", Journal of Business Research, vol. 64, n. 2, pp. 220-226.

BATRA R., SINHA I. (2000), "Consumer-level factors moderating the success of private label brands", Journal of Retailing, vol. 76, n. 2, pp. 175-191.

BERTOLI G., BUSACCA B., PELLONI O. (2008), "La valutazione di un'estensione di marca: consonanza percettiva, familiarità e reputazione", Sinergie, n. 77, pp. 45-65.

BOLTON R.N., DREW J.H. (1994), "Linking customer satisfaction to service operation and outcomes", in Oliver R.L., Rust R.T. (a cura di), Service Quality: New Directions in Theory and Practice, Sage, Thousand Oaks.

BOTTI S., SOSCIA I. (1999), "L'innovazione della distribuzione europea", in Valdani E., Castaldo S. (a cura di), Euromanagement. Scenari competitivi e politiche aziendali in un'Europa quasi compiuta, Egea, Milano.

BURT S. (2000), "The strategic role of retail brands in British grocery retailing", European Journal of Marketing, vol. 34, n. 8, pp. 875-890.

BURTON S., LICHTENSTEIN D.R., NETEMEYER R.G., GARRETSON J.A. (1998), “A scale for measuring attitude toward private label products and an examination of its psychological and behavioral correlates" Journal of the Academy of Marketing Science, vol. 26, n. 4, pp. 293-306.

BUSACCA B., BERTOLI G. (2012), "Celebrity endorsement, brand extension, brand loyalty", Sinergie, n. 89, pp. 51-67 
CASTALDO S., MAURI C. (2007), Innovazione, Experience, Partnership. Casi di innovazione nel retail, Franco Angeli, Milano.

COLGATE M., ALEXANDER N. (2002), "Retailers and diversification: the financial service dimension", Journal of Retailing and Consumer Services, vol. 9, n. 1, pp. 1-11.

CORSTJENS M., LAL R. (2000), "Building Store Loyalty through Store Brands", Journal of Marketing Research, vol. 37, n. 3, pp. 281-291.

COX D.R., SNELL E.J. (1989), The analysis of binary data, Chapman and Hall, London.

CRISTOL S.M., SEALEY P. (2000), Simplicity Marketing - End Brand Complexity, Clutter, and Confusion, The Free Press, New York.

CUNNINGHAM I.C.M., HARDY A.P., IMPERIA G. (1982), "Generic brands versus national brands and store brands", Journal of Advertising Research, vol. 22, n. 5, pp. $25-32$.

DAVIDSON W.R., BATES A.D., BASS S.J. (1976), “The retail life cycle concept”, Harvard Business Review, n. 54, pp. 89-96.

DAWSON J. (2001), "Is there a new commerce in Europe", The international Review of Retail, Distribution and Consumer Research, vol. 11, n. 3, pp. 287-299.

DEKIMPE M.G., STEENKAMP J.B.E.M., MELLENS M., ABEELE P.V. (1997), "Decline and variability in brand loyalty", International Journal of Research in Marketing, vol. 14, n. 5, pp. 405-420.

DE WULF K., ODEKERKEN-SCHRÖDER G., IACOBUCCI D. (2001), "Investments in consumer relationship: A cross-country and cross-industry exploration", Journal of Marketing, vol. 65, n. 4, pp. 33-50.

DICK A., JAIN A., RICHARDSON P. (1995), "Correlates of store brand proneness: some empirical observations", Journal of Product Brand Management, vol. 4, n. 4, pp. 1522.

DUPUIS M. (2001), L'innovation commerciale, Etudes et Recherche, Ceridice, Paris.

FILSER M. (1992), I canali della distribuzione, Milano, Etas.

GARRETSON J.A., FISHER D., BURTON S. (2002), "Antecedents of private label attitude and national brand promotion attitude: similarities and differences", Journal of Retailing, vol. 78, n. 2, pp. 91-99.

GIST R.R. (1968), Retailing, concept and decision, Wiley, New York.

GOLINELLI G.M. (2004), "Ridefinire il valore della marca”, Sinergie, n. 63, pp. 221-227.

GRAYSON K., AMBLER T. (1999), "The dark side of long term relationships in marketing services", Journal of Marketing Research, vol. 36, n. 1, pp. 132-141.

HOLLANDER S.C. (1960), "The wheel of retailing", Journal of Marketing, Vol. 25, n.1, pp. 37-42.

HOSMER D.W., LEMESHOW S. (2000), Applied logistic regression, Wiley, New York.

HUDDLESTON P., WHIPPLE J., VANAUKEN A. (2004), "Food store loyalty: Application of a consumer loyalty framework", Journal of Targeting, Measurement and Analysis for Marketing, vol. 12, n. 3, pp. 213-230.

KAHNEMAN D., TVERSKY A. (1973), "On the psychology of prediction”, Psychological review, vol. 80, n. 4, pp. 237-251.

KARRY S., ZACCOUR G. (2006), "Could co-op advertising be a manufacturer's counterstrategy to store brands?", Journal of Business Research, vol. 59, n. 9, pp. 10081015.

KELLER K.L. (1993), "Conceptualizing, measuring, and managing customer-based brand equity", Journal of Marketing, vol. 57, n. 1, pp. 1-17.

LAFORET S. (2007), "British grocers' brand extension in financial services", Journal of Product \& Brand Management, vol. 16, n. 2, pp. 82-97. 
LICHTENSTEIN D.R., RIDGWAY N.M., NETEMEYER R.G. (1993), "Price perceptions and consumer shopping behavior: a field study", Journal of Marketing Research, vol. 30, n. 2, pp. 234-245.

LUGLI G. (1993), Economia e politiche di marketing delle imprese commerciali, Utet, Torino.

LUGLI G. (1998), "I formati di punto vendita nel marketing commerciale e industriale", Trade Marketing, n. 23, pp. 47-92.

LUGLI G. (a cura di) (2003), Branding distributivo, Egea, Milano.

LUGLI G. (a cura di) (2009), Marketing distributivo. La creazione di valore nella distribuzione despecializzata, Wolters Kluver Italia, Milano.

MACINTOSH G., LOCKSHIN L.S. (1997), "Retail relationships and store loyalty: a multilevel perspective", International Journal of Research in Marketing, vol. 5, pp. 487-97.

MÄGI A.W. (2003), "Share of wallet in retailing: The effects of customer satisfaction, loyalty cards and shopper characteristics", Journal of Retailing, vol. 79, n. 2, pp. 97-106.

MANZUR E., OLAVARRIETA S., HIDALGO P., FARÍAS P., URIBE R. (2011), "Store Brand and National Brand Promotion Attitudes Antecedents", Journal of Business Research, vol. 64, n. 3, pp. 286-291.

MARTINELLI E. (2010), "I prodotti/servizi "Extra" nel rapporto insegna-cliente", Mercati $e$ Competitività, vol. 4, n. 4, pp. 145-163.

MARTINELLI E. (2012), Distributori grocery in convergenza. Esperienze a confronto, Franco Angeli, Milano.

MCNAIR M.P (1958), "Significant trends and developments in the postwar period", in Smith, A.B (Eds),Competitive Distribution in a Free, High Level Economy and Its Implication for the University, University of Pittsburgh Press, Pittsburgh, PA.

MENARD S. (1995), Applied logistic regression analysis, Sage, Thousand Oaks.

MYERS J.G. (1967), "Determinants of Private Brand Attitude", Journal of Marketing Research, vol. 4, n. 1, pp. 73-81.

MYERS R. (1990), Classical and modern regression with applications, Duxbury, Boston.

NAGELKERKE N.J.D. (1991), "A note on a general definition of the coefficient of determination", Biometrika, n. 78, pp. 691-692.

NOGALES A.F., SUAREZ M.G. (2005), "Shelf Space Management of Private labels: a Case study in Spanish Retailing", Journal of Retailing and Consumer Services, vol. 12, n. 3, pp. 205-216.

NUNNALLY J.C., BERNSTEIN I.H. (1994), Psychometric theory (3rd Ed.), McGraw-Hill, New York.

PASTORE A. (1996), I nuovi rapporti tra Industria e Distribuzione, Cedam, Padova.

PASTORE A., FORNARI E., CECCONI V. (2007), "Sviluppo e riposizionamento delle marche commerciali", Finanza, Marketing e Produzione, n. 2, pp. 61-86.

PAULWELS K., SRINIVASAN S. (2004), "Who Benefits from Store Brand Entry", Marketing Science, vol. 23, n. 3, pp. 364-390.

PARK W., MILBERG S., LAWSON R. (1991), "Evaluation of brand extensions: the role of product feature similarity and brand concept consistency", Journal of Consumer Research, vol. 18, n. 2, pp. 185-193.

PUTSIS JR W.P., DHAR R. (2001), "An empirical analysis of the determinants of category expenditure", Journal of Business Research, vol. 52, n. 3, pp. 277-291.

RICHARDSON P., DICK A., JAIN A. (1994), "Extrinsic and intrinsic cue effects on perceptions of store brand quality", Journal of Marketing, vol. 58, n. 4, pp. 28-36.

REICHHELD F. (1993), "Loyalty-Based Management", Harvard Business Review, vol. 71, n. 2, pp. 64-73. 
REICHHELD F., SASSER W.E.JR. (1990), “Zero Defections: Quality Comes to Services", Harvard Business Review, vol. 68, n. 5, pp. 105-111.

SETHURAMAN R. (2003), "Measuring national brands' equity over store brands", Review of Marketing Science, vol. 1, n. 2, pp. 1-26.

SIROHI N.E., MCLAUGHLIN W., WITTINK D.R. (1998), "A Model of Consumer Perceptions and Store Loyalty intentions for a Supermarket Retailer, Journal of Retailing, vol. 74, n. 2, pp. 223-245.

SPRANZI A. (1991), La distribuzione commerciale. Economia del commercio e politica commerciale, Franco Angeli, Milano.

SPROTT D.E., SHIMP T.A. (2004), Using product sample to augment the perceived quality of store brands, Journal of Retailing, vol. 80, n. 4, pp. 305-315.

STEENKAMP J.B.E.M., VAN HEERDE H., GEYSKENS I. (2010), "What makes consumers willing to pay a price premium for national brands over private labels?", Journal of Marketing Research, Vol. 47, n.6, pp. 1011-1024.

SYMPHONYIRIGROUP (2012), Le Private Label in Europa - 2012. Esiste un limite alla crescita, Ottobre.

VARALDO R., DALLI D. (1989), "Le relazioni strategiche tra Industria e Distribuzione", Sinergie, n. 19, pp. 13-48.

\section{Siti internet}

http://www.iriworldwide.eu

http://www.nielsen.com

http://plmainternational.com/en/private_label12_en.htm 